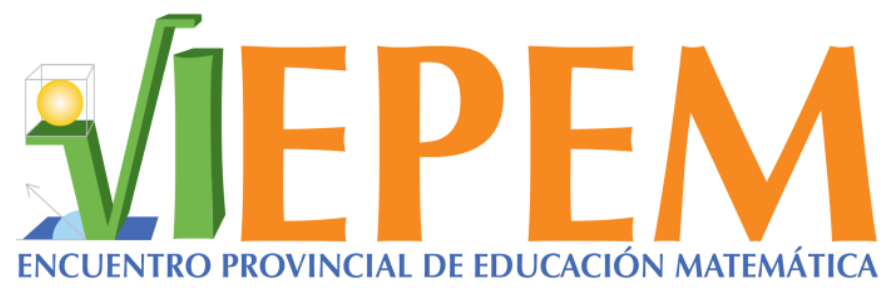

VI Encuentro Provincial de Educación Matemática

27 al 29 de setiembre, 2017. Puntarenas, Costa Rica

\title{
Algunos errores en matemática que cometen los estudiantes que ingresan a la universidad
}

\author{
Mario Castillo Sánchez \\ mario.castillo.sanchez@una.cr \\ Universidad Nacional \\ Costa Rica \\ Ronny Gamboa Araya \\ ronny.gamboa.araya@una.cr \\ Universidad Nacional \\ Costa Rica \\ Randall Hidalgo Mora \\ randall.hidalgo.mora@una.cr \\ Universidad Nacional \\ Costa Rica
}

\begin{abstract}
Resumen
En este documento se presentan algunos de los errores cometidos por los estudiantes durante la realización del examen de diagnóstico que aplicó la Escuela de Matemática de la Universidad Nacional a estudiantes de primer ingreso a la institución en el año 2017. Del total de exámenes se seleccionó una muestra aleatoria de 100 pruebas. Posteriormente, por cada ítem, se hizo una elección de las pruebas donde los estudiantes habían realizado algún procedimiento y la respuesta había sido incorrecta. En este documento se presentan únicamente algunos errores para las cinco preguntas donde hubo menor porcentaje de respuestas correctas. Los estudiantes presentan errores y dificultades al reconocer y aplicar conceptos relacionados con ángulos (suplementarios, complementarios, rectos, agudos), triángulos rectángulos y acutángulos y valor absoluto, entre otros. Además, presentan deficiencias para interpretar una situación y expresarla mediante una representación o dibujo. Es importante que el docente sea consiente que el estudio de los errores en matemática es una fuente de información para detectar no solo deficiencias sino para el planteamiento de estrategias orientadas al reforzamiento de los contenidos matemáticos, tanto en la secundaria como en la universidad.
\end{abstract}

Palabras clave: errores; matemática; estudiantes; universidad.

Ponencia

Castillo, M.; Gamboa, R.; Hidalgo, R. (2017). Algunos errores en matemática que cometen los estudiantes que ingresan a la universidad. En Y. Morales-López, M. Picado, R. Gamboa, C.

Martínez, M. Castillo y R. Hidalgo (Eds.), Memorias del VI Encuentro Provincial de Educación Matemática, Costa Rica, 2017 (pp. 69-82). Heredia: Universidad Nacional. ISBN: 978-9968-96615-3. DOI: http://dx.doi.org/10.15359/epem.6.17 


\section{Introducción}

La finalización de la educación secundaria representa para los estudiantes el término de una etapa académica y personal. Para algunos, el ingreso a la universidad representa un nuevo reto y genera una enorme expectativa.

Al finalizar la educación secundaria, específicamente en el área de matemática, los estudiantes han adquirido conocimientos, destrezas, habilidades $\mathrm{y}$ han aprendido procedimientos algorítmicos que sirven de base para su educación universitaria y para enfrentarse a los primeros cursos del área en sus respectivas carreras. Pero, a la vez, poseen dificultades que también se trasladan a su educación universitaria.

En este sentido, Franchi y Hernández (2004) apuntan que las dificultades que tienen los estudiantes se evidencian a través de sus errores, por lo que es importante reflexionar acerca de su significado y origen.

En la Escuela de Matemática de la Universidad Nacional, con el fin de determinar el nivel de conocimiento matemático que poseen los estudiantes de primer ingreso, se ha convocado cada año a dicha población para la aplicación de una prueba de conocimientos y destrezas en el área de matemática. Esta prueba se aplica desde el año 2008, sin carácter de obligatoriedad, y la población convocada son los estudiantes que ingresan por primera vez a carreras que contemplan en sus planes de estudio, al menos, un curso del componente matemático.

Esta prueba, corresponde a una estrategia mediante la cual se pretende recolectar información sobre una de las posibles causas de los altos niveles de deserción y reprobación que se presentan usualmente en los cursos de matemática: deficiencias en cuanto a conocimientos y destrezas en el área de la matemática con las que podrían estar ingresando a la universidad los estudiantes de primer ingreso (Escuela de Matemática, 2017, p.1).

Según se indica en el Informe de resultados examen de diagnóstico de Matemática: Estudiantes de primer ingreso del año 2017, en la prueba aplicada en enero de ese año, participaron 1694 estudiantes y se obtuvieron los siguientes resultados

a) Menos del $8 \%$ del total de estudiantes que realizaron la prueba, obtuvieron una nota superior a 60 .

b) Cerca del $50 \%$ de los estudiantes participantes de la prueba, obtuvieron calificaciones entre 20 y 40 . La nota promedio del total estudiantes que realizaron la prueba fue de 41,32 .

c) La nota promedio obtenida por los estudiantes participantes refleja una situación similar en cada una de las sedes en las que la prueba fue aplicada.

d) Se registraron menos estudiantes procedentes de instituciones educativas privadas con calificaciones inferiores a 40 que estudiantes procedentes de instituciones educativas públicas diurnas con calificaciones en este mismo rango.

e) El grupo de estudiantes participantes procedentes de instituciones educativas privadas es el que registra la mayor nota promedio. 
f) Se registran situaciones similares en cuanto a la distribución, por sexo, de las notas obtenidas.

g) Se registra que la nota promedio obtenida por los estudiantes participantes de sexo femenino es menor que la nota promedio obtenida por los estudiantes participantes de sexo masculino.

h) Se registran distribuciones similares en cuanto a las notas obtenidas por los estudiantes participantes de las diferentes carreras y sedes consideradas.

Los datos obtenidos en el año 2017 son similares a los que se alcanzaron en años anteriores. En el año 2016, 1832 estudiantes realizaron el examen de diagnóstico y se obtuvo que menos del $2,5 \%$ de los alumnos que realizaron la prueba alcanzaron una nota superior a 60, la mayoría de estudiantes $(76,8 \%)$ obtuvieron calificaciones entre 20 y 40 y el promedio de las calificaciones obtenidas fue de 29,73 (Escuela de Matemática, 2016).

En el 2015, por ejemplo, 1760 alumnos participaron en dicha prueba. El 76,53\% de los estudiantes (1347) obtuvo una nota que varió entre 20 a menos de 40. Solamente un $2,78 \%$ de los que aplicaron el examen (49 estudiantes) tuvieron como calificación una nota mayor a 60. El promedio de nota de toda la población fue de 29,2 (Escuela de Matemática, 2015).

Ante esta situación y con el propósito de identificar los errores cometidos por los estudiantes durante la realización de esta prueba, se realizó el análisis de una muestra de los exámenes resueltos por los estudiantes en año 2017. El por qué analizar estos errores se basa en que, tal como lo señala Mancera (2015), los errores demuestran no solo lo que no maneja o conoce el estudiante sino que proporcionan el punto de partida para establecer posibles causas de dicho equívocos.

Es evidente que los errores que posee un estudiante influyen en el aprendizaje de otros contenidos, por lo que es imprescindible conocerlos para concientizar a estos para su reconocimiento y que asuman la necesidad de superarlos para lograr un verdadero aprendizaje (Engler, Gregorini, Müller, Vrancken y Hecklein, 2004). Además de lo anterior, los autores señalan que el análisis de los errores sirve para ayudar al docente en la creación y organización de estrategias orientadas al logro de un mejor aprendizaje y la corrección de los errores, haciendo énfasis en aquellos aspectos que generan más dificultades para sus estudiantes.

\section{Marco teórico}

Existen diferentes aproximaciones de lo que es error. Incluso, algunos autores no se atreven a dar una definición. Sin embargo, con el propósito de dar una idea de algunas aproximaciones que se han encontrado en la literatura, se presentan las siguientes.

- "Hablamos de error cuando el alumno realiza una práctica (acción, argumentación, etc.) que no es válida desde el punto de vista de la institución matemática escolar" (Godino, Batanero y Font, 2004, p. 73).

- "Se podría definir "error" como un concepto equivocado o juicio falso. Por su parte, la equivocación se define como el tener o tomar una cosa por otra, juzgando u obrando desacertadamente" (Lucchini, Cuadrado y Tapia, 2006, p. 3). 
- "Se considera que el error es un conocimiento deficiente, insuficiente, imperfecto, defectuoso, escaso o incompleto; una desviación de un conocimiento establecido" (Carrión, 2007, p. 11).

Aunque no existe una definición única sobre lo que es un error, Mancera (2015) señala que el análisis de los errores cometidos por los estudiantes en su proceso de aprendizaje aporta información relevante sobre cómo se construye el conocimiento matemático y constituyen una importante herramienta para mostrar el estado del conocimiento en los alumnos, aspecto primordial para realimentar el proceso de enseñanza y aprendizaje.

En este sentido, el autor menciona que la regularidad de ciertos errores ha permitido su clasificación por varios autores, sin que ello sea una labor acabada, ya que las categorías presentadas por los autores no son excluyentes y rara vez un error obedece a una única causa (Mancera, 2015).

En el presente trabajo se utilizará la clasificación propuesta por Abrate, Pochulu y Vargas (2006), la cual señala varios tipos de errores

- Errores debidos al lenguaje matemático. Son producidos por una traducción incorrecta de hechos matemáticos descritos en un lenguaje natural a otro más formal en el lenguaje matemático, o de un lenguaje simbólico a otro simbólico distinto.

- Errores debidos a dificultades para obtener información espacial. Son atribuidos a deficiencias en la capacidad para pensar mediante imágenes espaciales o visuales llevando a interpretaciones incorrectas de información o hechos matemáticos.

- Errores debidos a inferencias o asociaciones incorrectas. Son generados por aplicar reglas y propiedades justificadas por esquemas similares o por inferir que son válidas en contextos parecidos o relacionados.

- Errores debidos a la recuperación de un esquema previo. Son causados por la persistencia de algunos aspectos del contenido o del proceso de solución de una situación aunque las condiciones fundamentales de la tarea matemática en cuestión se han modificado.

- Errores debidos a cálculos incorrectos o accidentales. Son errores que se presentan cuando cada paso en la realización de la tarea es correcto, o responde a la lógica interna del procedimiento esperado, pero el resultado final no es la solución debido a los errores de cálculo que se presentaron en la ejecución de operaciones básicas, o acarreados por la transferencia equivocada de símbolos y números involucrados en la situación.

- Errores eventuales debidos a deficiencias en la construcción de conocimientos previos. Son causados por aprendizajes incorrectos o inadecuados de hechos, destrezas y conceptos previos que interfieren en un adecuado procesamiento de la información.

- Errores debidos a la ausencia de conocimientos previos. Son causados por la carencia de aprendizajes de hechos, destrezas y conceptos previos, que inhiben totalmente el procesamiento de la información.

A partir de la clasificación anterior, se asume el concepto de dificultad como 
toda característica académica (en cuanto a lectura, escritura y matemáticas) que hace que el estudiante se muestre desnivelado con relación a sus compañeros de aula y al programa académico y a lo que se espera de él. Un individuo que tiene dificultad en el aprendizaje es aquella persona que no ha alcanzado un desarrollo integral en sus capacidades y por tanto tendrá problemas en el desenvolvimiento de las competencias básicas (Gómez, Wilches, Ruiz y Corrales, 2012, p.29).

Aunque se reconoce que no existe una clasificación única de los errores, la descrita anteriormente se tomó como base debido a que fue la empleada por dichos autores en una investigación orientada a analizar las dificultades y errores de conceptos y procesos matemáticos en una evaluación escrita de los alumnos al ingresar a la universidad.

Para ello los autores trabajaron con estudiantes aspirantes a ingresar a las carreras de Ciencias Económicas de la Universidad Nacional de Villa María, durante el año académico 2004, mientras cursaban el módulo de matemática del curso de ingreso. Los investigadores elaboraron un instrumento considerando los errores que detectan frecuentemente los profesores de matemática en el aprendizaje de los alumnos.

Según lo anterior, la población con la que los autores trabajaron presenta características similares con la nuestra, al ser estudiantes que iniciaban o estaban próximos a iniciar su vida universitaria.

Es importante señalar que los errores de un estudiante son la manifestación de un proceso complejo en el que interactúan muchas variables y actores: profesor, alumno, currículo, contexto sociocultural, entre otras, y que aún no se ha completado un desarrollo teórico sistemático para clasificar, interpretar, predecir y superar errores y dificultades en busca de un aprendizaje de calidad (Engler et al., 2004).

\section{Metodología}

El estudio realizado es de naturaleza cualitativa. Se enfatizó en la descripción de los errores cometidos por los estudiantes en la resolución del examen de diagnóstico que realizó la Escuela de Matemática en el año 2017. Esta prueba constaba de 60 ejercicios de selección única y contaban con tres horas para su realización. Los ejercicios propuestos en la prueba fueron sometidos, durante la elaboración de esta, a análisis de validación por medio de técnicas estadísticas. Para su resolución, los estudiantes podían hacer uso de la calculadora y debían realizar, en el folleto del examen, todas las anotaciones que le permitían resolver los ejercicios propuestos. Los ejercicios propuestos en la prueba abarcaban temas como funciones, trigonometría, factorización, fórmulas notables, números reales, radicación y potenciación, ecuaciones, entre otros.

Esta prueba la realizaron 1694 estudiantes (Escuela de Matemática, 2017). Con la base de datos donde se consignaban las respuestas de los alumnos, se analizó el porcentaje de respuestas correctas para cada ítem. Del total de exámenes se seleccionó una muestra aleatoria de 100 pruebas. Posteriormente, por cada ítem, se hizo una elección de las pruebas donde los estudiantes habían realizado algún procedimiento y la respuesta había sido incorrecta. Para estos casos se describió el error en el procedimiento y se clasificó según la tipificación realizada por Abrate, Pochulu y Vargas (2006). Es importante indicar, como ya se ha mencionado, que la clasificación de los errores no es única y excluyente, por lo que

un error puede estar asociado a distintos aspectos. El tipo de error identificado se relacionó 
según la descripción realizada y la definición dada por los autores antes mencionados. En este documento se presentan únicamente algunos errores para las cinco preguntas donde hubo menor porcentaje de respuestas correctas.

\section{Análisis}

Los siguientes ítems del examen de diagnóstico fueron los que menor porcentaje de respuestas correctas obtuvieron. Se describe, además, los conocimientos previos requeridos para dar solución a la pregunta planteada.

\begin{tabular}{|c|c|}
\hline Ítem & Conocimientos previos \\
\hline $\begin{array}{l}\text { 50. Suponga } \angle A B C \text { y } \angle M S T \text { son suplementarios, además } \angle A B C \text { y } \angle H F J \text { son com- } \\
\text { plementarios. Si } \angle M S T=110^{\circ} \text {, entonces } \angle H F J \text { es } \\
\text { a) } 20^{\circ} \\
\text { b) } 70^{\circ} \\
\text { c) } 90^{\circ} \\
\text { d) } 110^{\circ}\end{array}$ & $\begin{array}{ll}\text { - } & \text { Definición de ángulos suplementarios. } \\
\text { - } & \text { Definición de ángulos complementarios. } \\
\text { - } & \text { Resolución de un problema mediante el } \\
& \text { planteamiento de una ecuación lineal. } \\
\text { - } & \text { Resolución de ecuaciones lineales. }\end{array}$ \\
\hline $\begin{array}{l}\text { 3. Considere las siguientes proposiciones } \\
\text { I.) }|3-5|=-2 \\
\text { II.) }|5-3|=2 \\
\text { ¿Cuáles de ellas son verdaderas? } \\
\text { a) Ambas. } \\
\text { b) Ninguna. } \\
\text { c) Solo la I. } \\
\text { d) solo la II. }\end{array}$ & $\begin{array}{l}\text { - } \quad \text { Definición de valor absoluto. } \\
\text { - } \quad \text { Aritmética básica. }\end{array}$ \\
\hline $\begin{array}{l}\text { 39. Pedro tuvo un accidente en su bicicleta que le causó una herida de } 9 \mathrm{~cm}^{2} \text { en una } \\
\text { pierna. ¿Qué porcentaje aproximado de la herida cicatrizo luego de diez días? } \\
\text { a) } 1,83 \% \\
\text { b) } 6,70 \% \\
\text { c) } 93,3 \% \\
\text { d) } 98,2 \%\end{array}$ & $\begin{array}{l}\text { - Resolución de un problema mediante el } \\
\text { planteamiento de una función exponencial. } \\
\text { - } \quad \text { Cálculo de porcentajes. }\end{array}$ \\
\hline $\begin{array}{l}\text { 49. Considere un triángulo rectángulo } \triangle A B C \text {, recto en } B \text {. Si } P \text { es el punto medio de } \\
\overline{B C} \text { y } Q \text { es un punto en } A B \text { tal que } B Q=2 A Q \text {, entonces el cociente entre el área } \\
\text { del } \triangle A Q C \text { y el } \triangle P Q C \text { es } \\
\text { a) } \frac{1}{2} \\
\text { b) } 1 \\
\text { c) } 2 \\
\text { d) } \frac{2}{3}\end{array}$ & $\begin{array}{ll}\text { - } & \text { Definición de un triángulo rectángulo. } \\
\text { - } & \text { Definición de punto medio. } \\
\text { - } & \text { Área de un triángulo. }\end{array}$ \\
\hline $\begin{array}{l}\text { Considere la información del siguiente cuadro para responder las preguntas } 58,59 \text { y } 60 \text {. } \\
\qquad \begin{array}{l}\text { El conductor de un automóvil observa con un ángulo de elevación de } 30^{\circ} \text { el } \\
\text { punto más alto de un edificio. Conduce en línea recta } 300 \mathrm{~m} \text { hacia la base del } \\
\text { edificio y vuelve a observar el punto más alto, con un ángulo de elevación de } \\
60^{\circ} \text {. }\end{array}\end{array}$ & - $\quad$ Definición de ángulo de elevación y \\
\hline $\begin{array}{l}\text { 58. Determine la distancia entre el primer punto de observación y la parte más alta del } \\
\text { edificio. } \\
\text { a) } 173,20 \mathrm{~m} \\
\text { b) } 519,61 \mathrm{~m} \\
\text { c) } 600 \mathrm{~m} \\
\text { d) } 346,41 \mathrm{~m}\end{array}$ & $\begin{array}{l}\text { - } \quad \begin{array}{l}\text { Razones trigonométricas en un triángulo } \\
\text { rectángulo. }\end{array} \\
\text { - Resolución de problemas mediante el } \\
\text { planteo de ecuaciones trigonométricas. }\end{array}$ \\
\hline
\end{tabular}


Respecto a los errores cometidos por los estudiantes al resolver las preguntas planteadas, a continuación se describen algunos de los errores presentados.

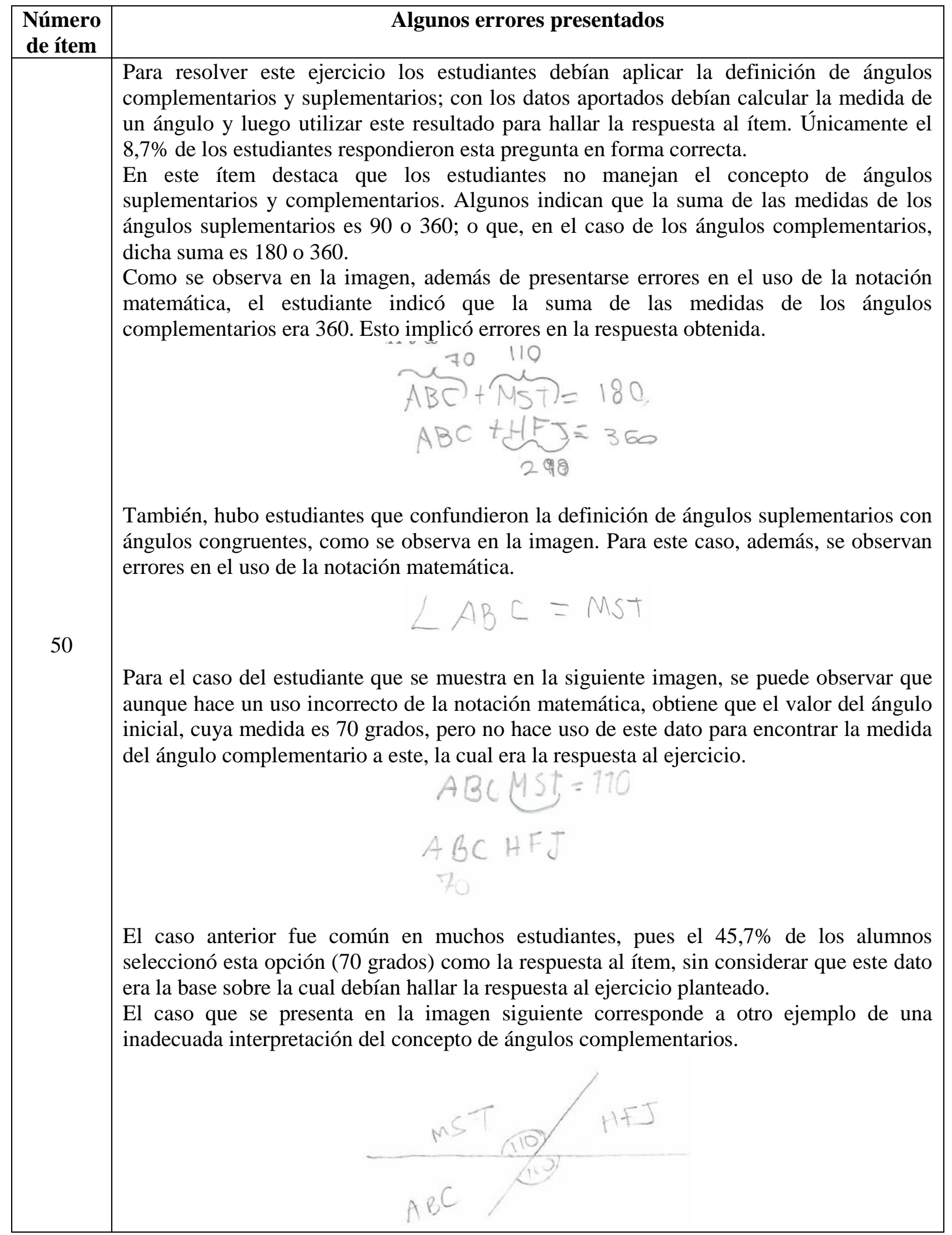




\begin{tabular}{|c|c|}
\hline & Algunos errores presentados \\
\hline & $\begin{array}{l}\text { Aunque la representación realizada por el estudiante es correcta con respecto a los ángulos } \\
\text { suplementarios, los ángulos complementarios fueron representados como opuestos por el } \\
\text { vértice, lo cual no se adecuaba a los datos presentados en el ejercicio. } \\
\text { Los estudiantes también tienden a particularizar ciertos conceptos. Por ejemplo, aunque en } \\
\text { el ejercicio se hacía referencia a dos ángulos suplementarios en general, el dibujo } \\
\text { realizado por un estudiante representa un caso particular, pues ambos son dibujados como } \\
\text { ángulos rectos. } \\
\text { Lo anterior llevó a un error y una contradicción con los datos mostrados en el ejercicio, } \\
\text { pues según el enunciado uno de los ángulos dibujados por el estudiante como ángulo recto } \\
\text { era complementario con otro, lo cual resultaba incorrecto según su representación. } \\
\text { En este ejercicio en particular, se visualiza que los estudiantes no poseen adecuadas } \\
\text { estrategias para la revisión de sus procedimientos, pues no interpretan sus } \\
\text { representaciones ni los datos obtenidos según el contexto del ejercicio y si estos se } \\
\text { adecúan a él o no. } \\
\text { Tipos de errores } \\
\text { - Errores debido al lenguaje matemático, pues se no se hace un uso adecuado de la } \\
\text { notación matemática. } \\
\text { - Errores eventuales debidos a deficiencias en la construcción de conocimientos previos, } \\
\text { pues no se identifican los conceptos de ángulos complementarios y suplementarios. } \\
\text { - Errores debidos a la recuperación de un esquema previo, pues aplican conceptos } \\
\text { erróneos en temáticas que se encuentran relacionadas como ángulos complementarios, } \\
\text { suplementarios, congruentes y opuestos por el vértice; además, particularizan las } \\
\text { definiciones a casos específicos. } \\
\text { - Errores debido a cálculos incorrectos o accidentales, pues el resultado final no es el } \\
\text { esperado debido a que no se completa el procedimiento para hallar la respuesta del ítem. }\end{array}$ \\
\hline 3 & $\begin{array}{l}\text { En este ejercicio los estudiantes no debían realizar ningún procedimiento. Solo debían } \\
\text { realizar la resta de números enteros que se especificaba en el ítem y aplicar el concepto de } \\
\text { valor absoluto de un número real. } \\
\text { Sin embargo, únicamente el 14,9\% respondió correctamente esta pregunta. El 77,5\% } \\
\text { marcó como respuesta la opción Ambas (el número tanto negativo como positivo). } \\
\text { Lo anterior refleja un inadecuado manejo del concepto de valor absoluto. } \\
\text { Tipos de errores } \\
\text { - Errores eventuales debidos a deficiencia en la construcción de conocimientos previos, } \\
\text { pues no se visualiza un aprendizaje correcto del concepto de valor absoluto. } \\
\text { - Errores debido a la ausencia de conocimiento previo, pues en algunos casos los } \\
\text { estudiantes no sabían qué significaba la notación de valor absoluto. }\end{array}$ \\
\hline 39 & $\begin{array}{l}\text { Para resolver el ejercicio planeado los estudiantes debían realizar una sustitución para } \\
\text { calcular el área cicatrizada y luego calcular el porcentaje que dicha área presentaba del } \\
\text { área original. } \\
\text { Este ejercicio fue respondido correctamente por } 17,1 \% \text { de los estudiantes. }\end{array}$ \\
\hline
\end{tabular}




\begin{tabular}{|c|c|}
\hline $\begin{array}{l}\text { Número } \\
\text { de ítem }\end{array}$ & Algunos errores presentados \\
\hline & 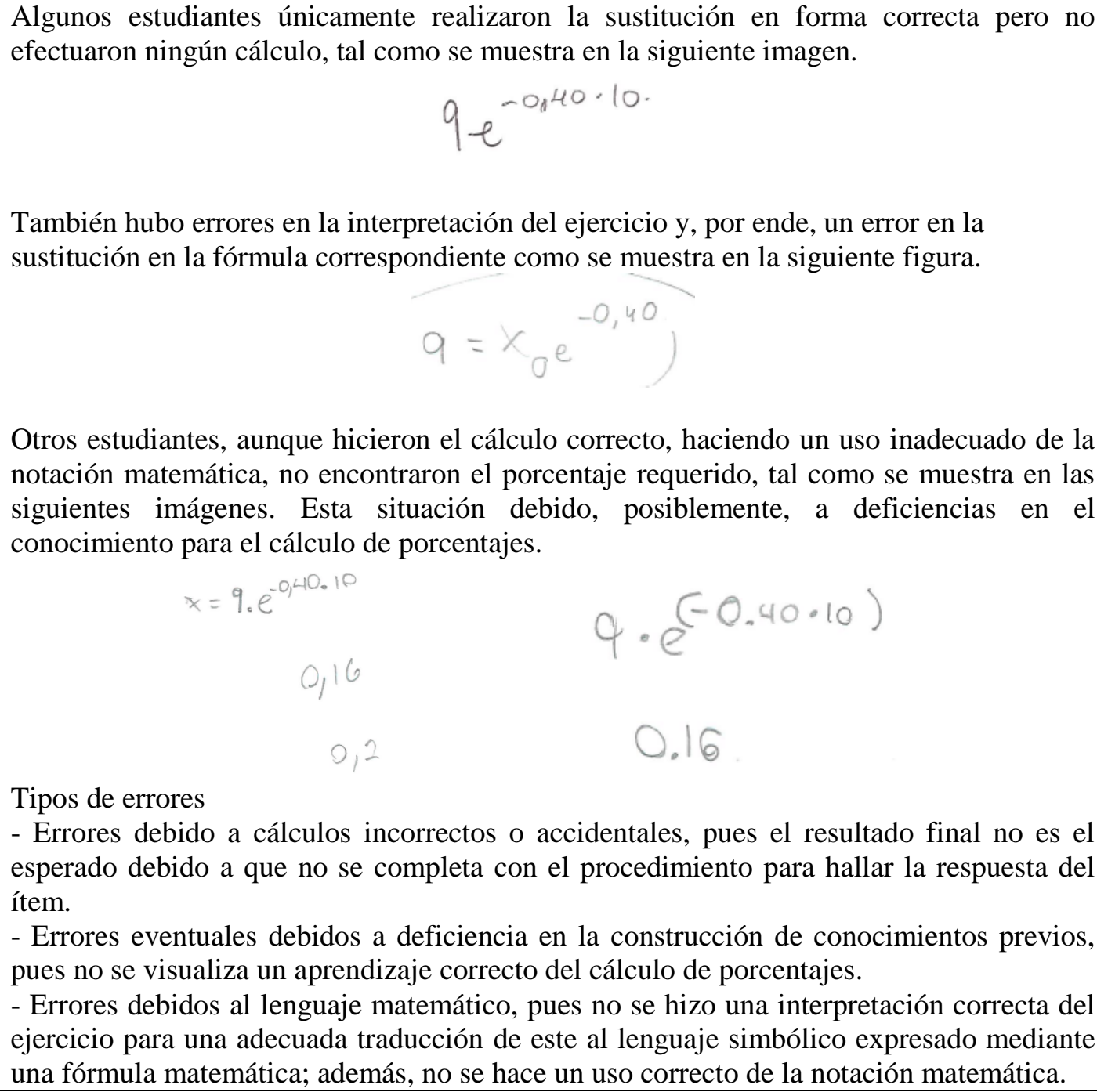 \\
\hline 49 & $\begin{array}{l}\text { En este ítem los estudiantes debían aplicar conceptos como triángulo rectángulo, ángulo } \\
\text { recto, punto medio, cociente, área de un triángulo, entre otros. Además, debían aplicar su } \\
\text { capacidad de visualización para interpretar el ejercicio y construir una representación de } \\
\text { este. } \\
\text { El porcentaje de respuesta correcta (opción b) fue de } 17,1 \% \text {. El porcentaje de respuestas } \\
\text { para las opciones a), c) y d) fueron, respectivamente, } 23,7 \% ; 34,5 \% \text { y } 23,7 \% \text {. } \\
\text { La dificultad más común fue la incorrecta interpretación del ítem y su representación. En } \\
\text { la siguiente figura se observa, por ejemplo, una incorrecta representación del ángulo recto, } \\
\text { pues se dibuja como un ángulo agudo. En este caso, el estudiante no realizó ningún otro } \\
\text { procedimiento. }\end{array}$ \\
\hline
\end{tabular}




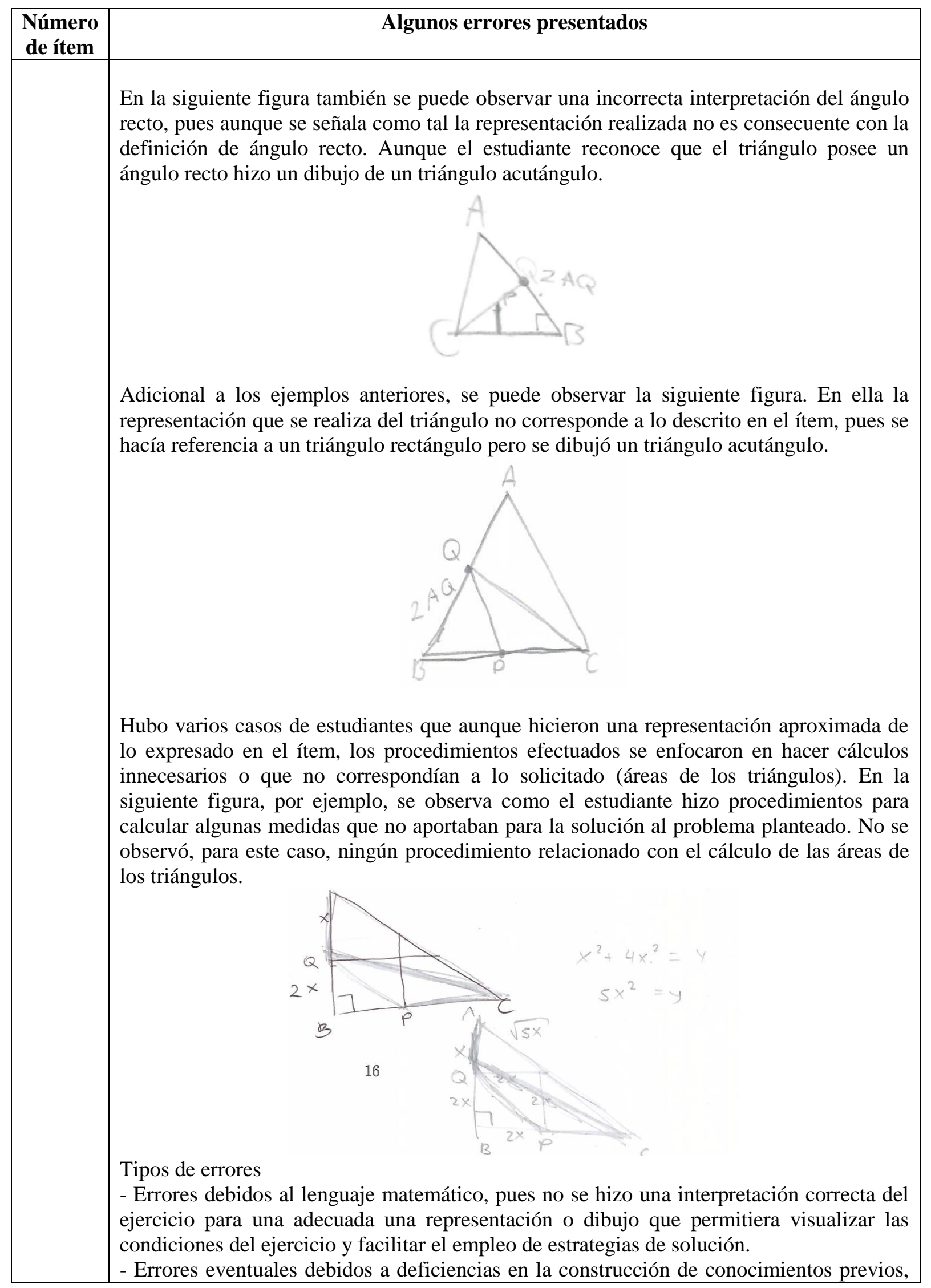

VI Encuentro Provincial de Educación Matemática, 


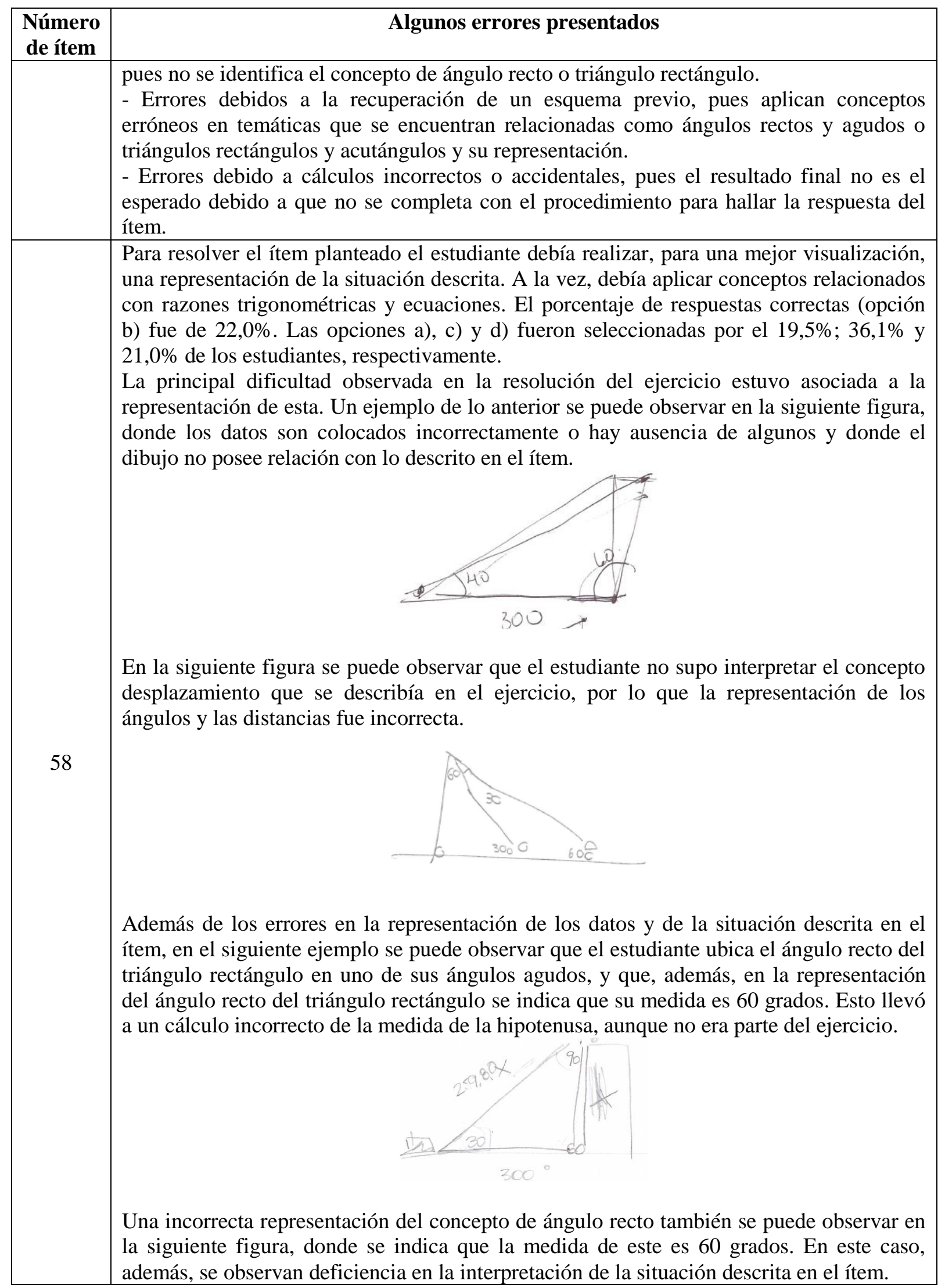




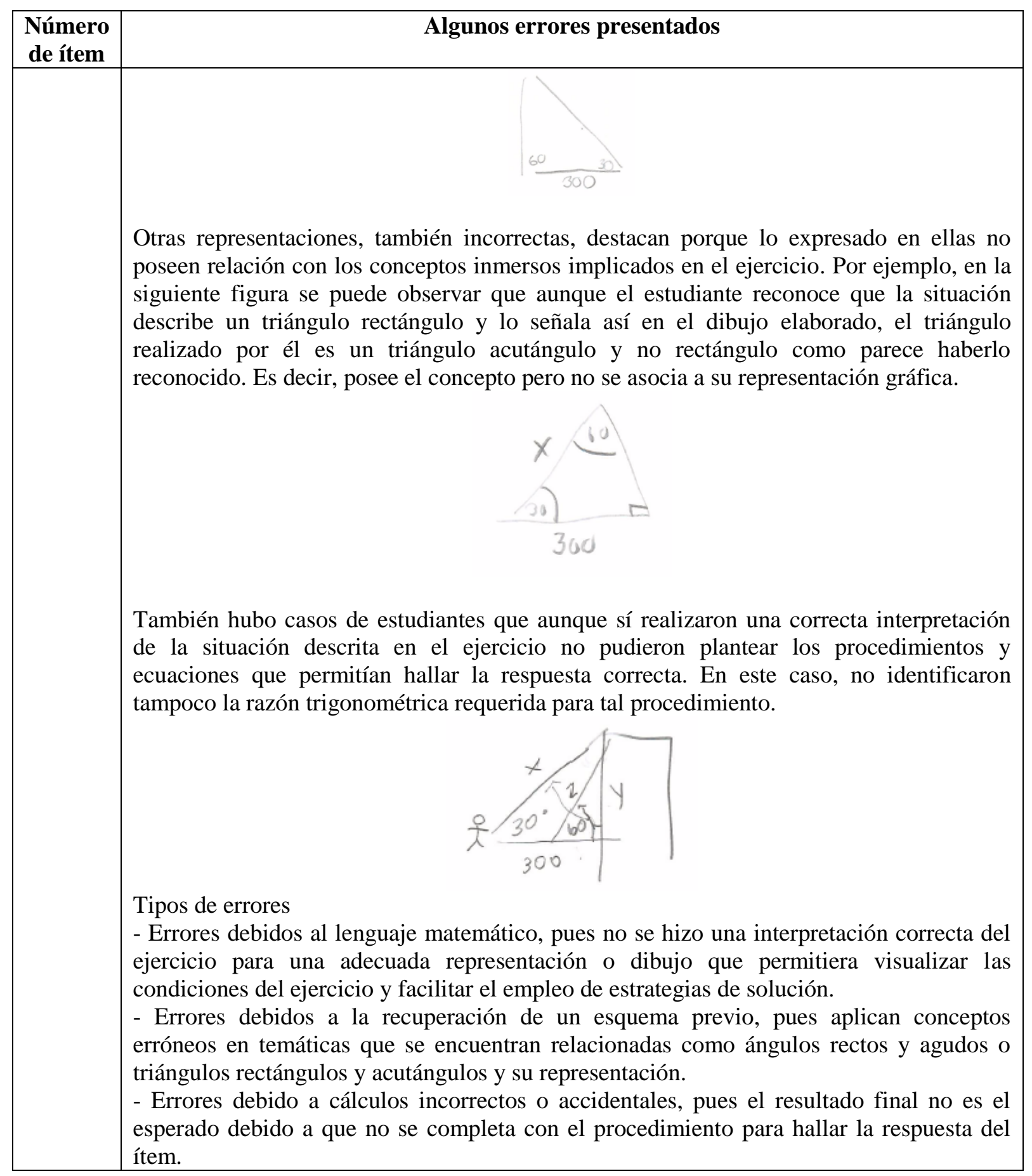




\section{Conclusiones}

El estudio de los errores debería ser un eje central en el proceso de enseñanza y aprendizaje de la matemática, ya que le permite al docente identificar los conceptos o procedimientos en los que el estudiante posee deficiencias o no ha logrado la comprensión adecuada.

El docente, además de calificar una prueba o ejercicio y determinar si este es correcto o incorrecto y asignar una nota, debe analizar los tipos de errores cometidos e identificar en cuáles aspectos el estudiante requiere apoyo. Se debe partir del hecho que con base en los conocimientos que posee el alumno se crean otros más complejos y que el error en el aprendizaje de unos impacta en la construcción de los otros.

Identificar y analizar los errores que cometen los estudiantes le permite al docente generar información valiosa sobre las dificultades de estos y utilizarla para crear estrategias orientadas a corregir los errores, solventar deficiencias, consolidar los conocimientos previos requeridos para la construcción de otros más complejos e implementar estrategias orientadas a su reforzamiento.

La idea del error como elemento para el castigo o censura debe ser cambiada por el docente y debe estimular al estudiante para aprovechar este como indicador de su aprendizaje.

\section{Reconocimiento}

Documento elaborado en el marco del proyecto de investigación: La deserción y repitencia en el curso de Matemática General en la Universidad Nacional, código SIA 0250-15, Universidad Nacional, Costa Rica.

\section{Referencias}

Abrate, R., Pochulu, M., y Vargas, J. (2006). Errores y dificultades en Matemática: Análisis de causas y sugerencias de trabajo. Buenos Aires, Argentina: Universidad Nacional de Villa María.

Carrión, V. (2007). Análisis de errores de estudiantes y profesores en expresiones combinadas con números naturales. Unión Revista Iberoamericana de Educación Matemática, 11, 19-57. Recuperado de http://www.fisem.org/www/union/revistas/2007/11/Union_011_007.pdf

Engler, A., Gregorini, M. I., Müller, D., Vrancken, S., y Hecklein, M. (2004). Los errores en el aprendizaje de matemática. Revista Premisas, (23), 23-29. Recuperado de http://soarem.org.ar/Documentos/23 Engler.pdf

Escuela de Matemática. (2015). Informe de resultados examen de diagnóstico de matemática: Estudiantes de primer ingreso del año 2015. Universidad Nacional. Documento no publicado.

Escuela de Matemática. (2016). Informe de resultados examen de diagnóstico de matemática: Estudiantes de primer ingreso del año 2016. Universidad Nacional. Documento no publicado. 
Escuela de Matemática. (2017). Informe de resultados examen de diagnóstico de matemática: Estudiantes de primer ingreso del año 2017. Universidad Nacional. Documento no publicado.

Franchi, L., y Hernández, A. I. (2004). Tipología de errores en el área de la geometría plana. Educere, 25, 196-204. Recuperado de http://www.saber.ula.ve/bitstream/123456789/19840/1/articulo10.pdf

Godino, J., Batanero, C. y Font, V. (2004). Fundamentos de la enseñanza y aprendizaje de la Matemática para maestros. Universidad de Granada, España. Recuperado de http://www.ugr.es/ jgodino/edumat-maestros/manual/9_didactica_maestros.pdf

Gómez, K., Wilches, L., Ruiz, R., y Corrales, Z. (2012). Dificultades en el aprendizaje de las matemáticas en los estudiantes del $\sigma^{\circ}$ grado de educación básica secundaria en la institución educativa Almirante Colón (Tesis de Licenciatura). Universidad Francisco de Paula $\quad$ Santander. Recuperado de http://190.242.62.234:8080/jspui/bitstream/11227/226/1/proyecto_unicartagena.pdf

Lucchini, G., Cuadrado, B., y Tapia, L. (2006). Errar no es siempre un error. Fundación Educacional Arauco (Fundar). Santiago, Chile. Recuperado de http://www.fundacionarauco.cl/_file/file_3878_errar\%20no\%20es\%20siempre\%20un \%20error.pdf

Mancera, E. (2015). Errar es un placer: El uso de los errores para el desarrollo del pensamiento matemático. 2a. ed. México: 3D Editorial.

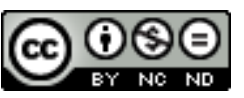

Esta obra está bajo una licencia de Creative Commons Reconocimiento-NoComercialSinObraDerivada 4.0 Internacional. 